\title{
Accessing Grid and Cloud Services Through a Scientific Web Portal
}

\author{
Marco Bencivenni - Diego Michelotto • Roberto Alfieri • Riccardo Brunetti • \\ Andrea Ceccanti • Daniele Cesini • Alessandro Costantini • Enrico Fattibene · \\ Luciano Gaido • Giuseppe Misurelli • Elisabetta Ronchieri • Davide Salomoni • \\ Paolo Veronesi • Valerio Venturi • Maria Cristina Vistoli
}

Received: 30 October 2013 / Accepted: 3 September 2014 / Published online: 23 September 2014

(C) Springer Science+Business Media Dordrecht 2014

\begin{abstract}
Distributed Computing Infrastructures have dedicated mechanisms to provide user communities with computational environments. While in the last decade the Grid has demonstrated to be a powerful paradigm in supporting scientific research, the complexity of the user experience still limits its adoption by unskilled user communities. Command line interfaces, X.509 certificates, template customization for job submission and data access tools require end-users to dedicate significant learning effort and thus represent a barrier to access Grid computing facilities. In this paper, we present a Web portal that solves the aforementioned limitations by providing simplified
\end{abstract}

Valerio Venturi deceased 25 December 2013

\section{R. Alfieri}

INFN Parma and Parma University,

Viale G.P. Usberti 7/A, Parma, Italy

M. Bencivenni - A. Ceccanti - D. Cesini - E. Fattibene -

D. Michelotto · G. Misurelli · P. Veronesi · V. Venturi

IGI and INFN-CNAF, Via Ranzani 13/2, Bologna, Italy

R. Brunetti · L. Gaido ( $\square)$

IGI and INFN Torino, Via P. Giuria 1, Torino, Italy

e-mail: luciano.gaido@to.infn.it

\author{
A. Costantini \\ IGI and INFN-Perugia, Via A. Pascoli, Perugia, Italy \\ E. Ronchieri · D. Salomoni - M. C. Vistoli \\ INFN-CNAF, Viale Berti Pichat 6/2, Bologna, Italy
}

access to Grid and Cloud computing services. The portal provides a set of interfaces that support federated authentication mechanisms, storage discovery and job description templates, enabling user communities to run specific use cases. We developed the portal framework within the Italian Grid Infrastructure where the major national user representatives drove its design, the implemented solutions and its validation by testing some specific use cases.

Keywords Web portal · Grid · Cloud · IGI · Digital Certificates

\section{Introduction}

In the last decade, distributed computing infrastructures (DCIs) have supported experimental research for heterogeneous communities (e.g., high energy physics and bioinformatics) by mostly exploiting the Grid paradigm [1]. The Worldwide LHC Computing Grid (WLCG) [2], one of the largest DCIs, has provided scientists with access to virtually unlimited computing power and storage, increasing the quality and throughput of their statistical data analysis and leading to the timely discovery of the Higgs Boson [3]. While the Grid is helping these communities in fulfilling their objectives, it still requires from the end-users significant effort and low-level technical knowledge to fully leverage its services. Our experience shows that it is often hard for untrained user communities to port their 
applications to the Grid environment due to the inherent complexity of its authentication mechanisms and job submission and data access interfaces.

As a consequence, several Web portals and science gateways [4-9] are being developed to hide the aforementioned complexities and simplify access to Grid services. Some existing solutions $[6,10]$ are domainspecific and typically rely on ROBOT certificates for user authentication [11]. The main drawback of this approach is that robot certificates hide whole user communities behind a small set of credentials, thus limiting the granularity and effectiveness of the Grid middleware tracing and authorization mechanisms. Furthermore, Grid security policies restrict the use of robot certificates to the submission of computational jobs that run only pre-determined applications. Hence, this approach is unsuitable for portals that need to support heterogeneous user communities. Other solutions, such as the Web Services Parallel Grid Runtime and Developer Environment (WS-PGRADE) [12], are not tied to a single community and allow users to access computing and storage services through a Web interface.

This paper describes the Italian Grid Infrastructure (IGI) [13] Web Portal, designed and developed to provide simplified access to Grid and Cloud resources to wide-ranging scientific communities. The portal provides customized user interfaces and support for federated authentication mechanisms, storage discovery and job description templates; it is designed to be usable in the context of different DCIs. None of the existing Web portals and Science Gateways available at the time of this writing satisfied all the above requirements or were adaptable to the needs of our user communities. In order to reduce the development effort and provide a robust solution, the portal glues together several existing components such as as MyProxy [14], WS-PGRADE, Distributed Infrastructure with Remote Control (DIRAC) [15], and Grid User Support Environment (gUSE) [16]. The portal Grid components are already in production, whereas the Cloud ones are in a prototypical evolving form. Since May 2013, the portal has supported 116 users, distributed in 12 VOs, who have submitted $17.8 \mathrm{k}$ jobs via DIRAC and $3.4 \mathrm{k}$ jobs via WS-PGRADE for a total amount of about 380k CPU-hours.

The paper is organized as follows. Section 2 presents related work which is classified according to the requirements of our user communities. Section 3 provides a high-level overview of the portal architecture, highlighting the authentication and authorization components in Section 4 and focusing on the computing and storage services in Section 5. Section 6 presents a set of use cases selected to validate the portal. Finally, Section 7 concludes the paper and presents directions for future work.

\section{Related Works}

Grid-enabled Web portals and science gateways have proved to be effective mechanisms for exposing computing resources and distributed systems to user communities without forcing them to deal with the complexities of the underlying systems. For this reason, several science collaborations and projects have developed their own Web application [4-9]. Even if these Web applications and portals have requirements similar to most known customer-oriented portals (typically services that include support for login, job submission and file management operations), they became domain-specific oriented applications adopting solutions that are difficult to be exported to other disciplines.

In light of these limitations, we developed the IGI portal by adopting and integrating specific technological solutions able to provide general and customizable Web interfaces. The portal aims to support smart users, who belong to different scientific domains, during the definition of their jobs, the submission of their applications and the exploitation of tools for their needs. This is what distinguishes our solutions from others that, like science gateways, tend to supply customized solutions by offering a limited set of operations. Although the literature reports many projects for accessing distributed resources through Web portals and gateways [12, 17-19], we devoted our attention to those supporting three elements: federated authentication, storage discovery, and job template. They cover most of the Grid activity scenarios such as granting federated access, customizing templates for job submission and locating storage endpoints for data replication. We focused on each element by selecting a subset of solutions, assessing their weaknesses and strengths, and also considering the requirements from our supported communities (described in Table 1). 
Table 1 The requirements collected from our user communities

\begin{tabular}{ll}
\hline Services & Requirements \\
\hline Authentication & Single-sign-on based authentication \\
and Authorization & $\begin{array}{l}\text { Personal certificate handling } \\
\text { Certificate provisioning on demand }\end{array}$ \\
Workload Management & $\begin{array}{l}\text { Managing workflows } \\
\text { Detailing Job Description Language } \\
\end{array}$ \\
& (JDL) customization \\
& Getting customized Web interface \\
& applications \\
Dimplified access to data storage & Simplified data moving
\end{tabular}

Concerning the federated authentication based on online Certificate Authority (CA), we considered the following solutions:

GridCertLib [20] uses a short lived credential service that issues certificates lasting maximum 11 days, hence the job lifetime must comply with this limitation. This is one of the reasons why we decided to discard GridCertLib in favour of solutions that support long-lived credentials (i.e. a 13-months validity) to address the lifetime constraint.

The CILogon [21] solution provides two main types of long-lived certificates each one with a specific level of assurance [22]: openID - the lowest, silver - the highest. While the openID relies on an authentication mechanism (e.g. Google account) unacceptable for the organisation coordinating the trust fabric for e-Science in Europe (EUGridPMA [23]), the silver, although accepted by EUGridPMA, only covers members of the InCommon federation [24], therefore excluding our referential research communities.

The Terena Certificate Service (TCS) [25] allows users to obtain their X.509 credentials by using a specific procedure: users must authenticate themselves to the Terena portal by using their federated identities, and obtain their X.509 certificates registered in the adopted browsers. Then users need to request a certificate and manage a key pair to upload their credentials into the IGI portal. TCS herein requires users to handle their certificates.

Due to the limitations of all the three solutions, we started designing our Web portal on top of an
Enterprise Java Based PKI Certificate Authority (EJBCA) [26] software that provides an EJBCA Web service and programmatic interfaces for different certificates signing request formats.

Referring to the storage discovery, we focused on the existing solutions to manage files that match specific file features, such as searching, drag and drop, and sharing:

Pydio [27] is based on a modular architecture allowing new portals to integrate external services and support further protocols by just developing plugins. In our experience, it has proven to be scalable in complex scenarios and supports multi-data and multi-users handling with suitable performances.

The ownCloud [28] framework could be a valid alternative to Pydio, but we discovered some weaknesses about scalability and performance.

The elFinder [29] solution provides PHP and Python connectors for portals binding, but the Java one is under development determining an important limitation for the Java-based portlets.

Consequently, we chose Pydio as file manager tool within the IGI Web portal. Since most recent browsers are compatible with HTML-5 and handle chunking operation through HTML-5 directly, we decided to adopt jQuery File Upload [30] for the file uploading.

The job submission was another important task to tackle. In particular, we had to consider that our primary research community - high energy physics uses its own middleware. For that reason we took DIRAC [15] for the following main reasons: it provides standard components with a well-defined set of extensions provided by the LHCb experiment [31]; it is also effective in sustaining multiple small groups access and supports code refurbishing, too. The IGI Web portal leverages DIRAC to offer general job submission services to a variety of scientific communities. Furthermore, it provides users with templates for customizing job description.

\section{Architecture}

We designed the portal architecture with the aim to reuse existing and maintained open solutions for all the features mentioned in Section 1. In addition, we adopted Liferay [32] - a popular open source portal and collaboration software made of functional 
units called portlets - to ensure a modular Web portal structure. The portlets are components that build dynamic Web contents and use the JSR168 [33] and JSR286 [34] standards to simplify portlets migration. We implemented portlets for each specific service (e.g. user authentication, job submission, and data management). This combination makes it easy to enrich the portal with new functionalities.

The IGI Web portal architecture, presented in Fig. 1, can be conceptually divided into five main layers. Each one contains components internally developed for the portal as well as provided by third-parties. Let us start describing the Fig. 1 content.

At the highest level, the Portal AuthN (Authentication) and AuthZ (Authorization) Layer verifies all the mandatory credentials provided by the users: X.509 certificate, Virtual Organization (VO) membership and Identity Provider (IdP) trusted by the portal. The certificate univocally identifies users and ensures a secure communication across the distributed resources. The AuthN portlet interfaces with singlesign-on solutions such as IdPs federations and the portal IdP. The AuthZ portlet collects users' credentials during the first access to the portal. For these two portlets, we added a MySQL-based DataBase (DB) [35], called portal DB, to the default Liferay DB to collect users' information. Not only does it store users' personal information (such as name, surname and mail), but also data concerning personal certificate and VOs membership.

At the second level, the External AuthZ and AuthN Services Layer supports the upper level in a set of operations:

- Users' credentials vetting leverages the IdPs federations and VOMSes (VO Membership Service) [36] components invoking single-sign-on or membership-credential requests.

- Providing missing credential exploits the portal IdP, online CA, and VOMS components acting as fallback and supplying an alternative credential chain.

- Storing delegated credentials (proxies) makes use of the MyProxy [14] components that manage short and long proxies.

In addition, the CA-bridge component is paramount for the interconnection among the AuthZ portlet and the other components involved in this layer,

Fig. 1 The IGI Web portal architecture: rectangular shapes identify internally developed components, rounded rectangular shapes show third-party components with local configuration, and finally oval shapes represent third-party components used as they are

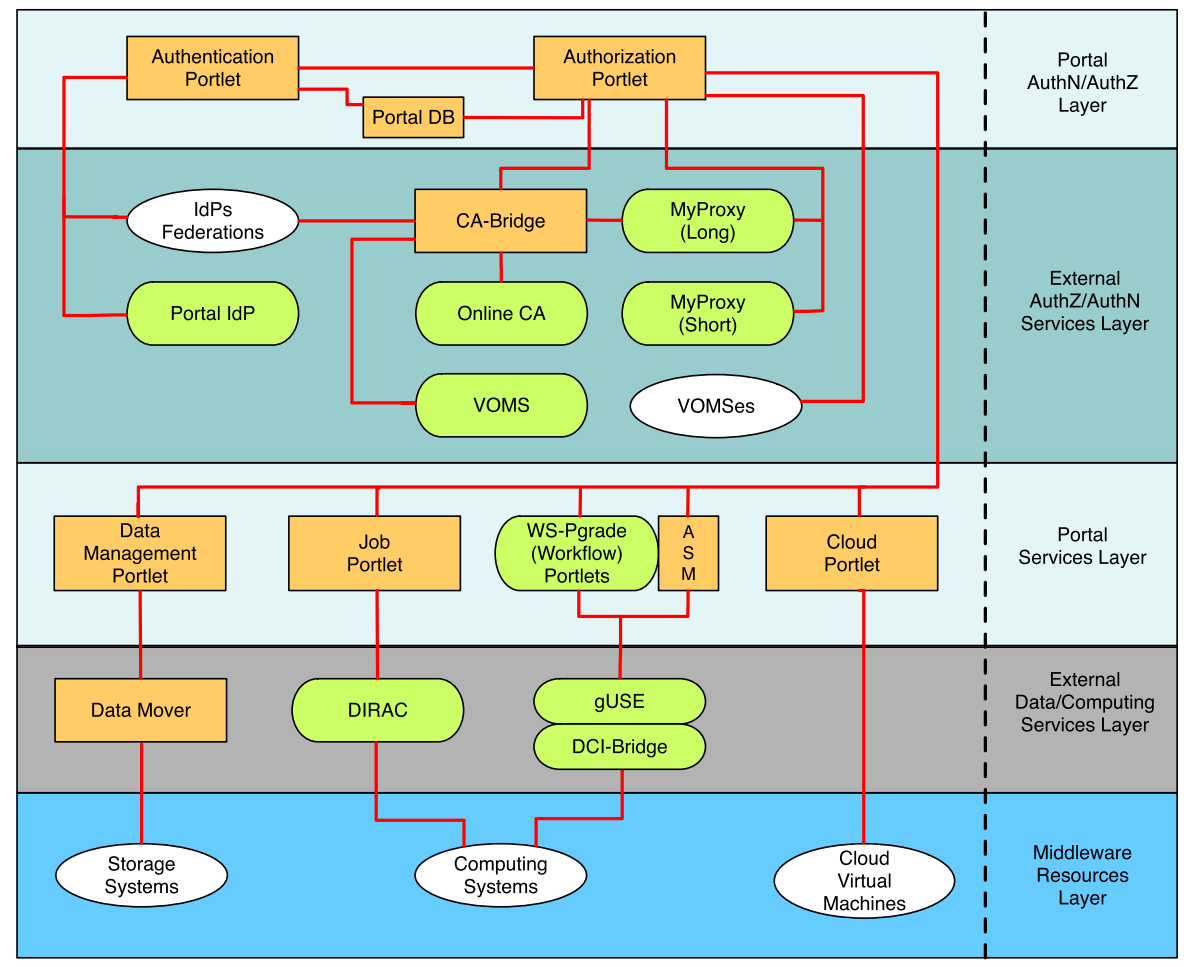


performing all the necessary steps to validate users identity, to provide a X509 certificate, to generate the Grid credentials and to archive user data. The CAbridge, MyProxy server and online CA components implement a certificate provisioning service integrated in the portal framework. For the reasons detailed in Section 2 the online CA supplies MICS (Member Integrated Credential Services) certificates [37] with a 13-months validity.

At the middle level, the Portal Services Layer contains all the portlets implementing data management, job submission, application and workflow submissions, and cloud provisioning. While a job is a sequence of key pairs (attribute, value) based on the JDL, a workflow is a sequence of connected jobs where the execution of one or more steps depend on the results of the previous ones. WS-PGRADE consists of various portlets (following the Liferay supported standards) providing different functionalities ranging from user registration to data management up to workflow handling; however, we only used the workflow feature. In order to hide the complexity of workflow configuration and execution to specific communities, we developed different portlets based on the Application Specific Management (ASM) APIs, available in the WS-PGRADE framework. These portlets provide application-specific interfaces and use the workflows defined in WS-PGRADE. Both WSPGRADE and ASM interact with the Grid and Cloud user support environment (gUSE). The Job portlet allows the portal to submit jobs over computing systems, while the Cloud portlet enables an interface towards user-specified virtual machines in an Infrastructure as a Service scenario. At last the Data Management portlet interfaces with the storage systems. This level allows to add custom Web interfaces just by creating new portlets to support new applications, providing a set of scenarios suitable for users' needs.

At the second to last level, the External Data and Computing Layer provides the tools to handle data required by the portlets in the Portal Services Layer. The Data Mover component allows users to transfer data among Grid resources, hiding all the complexity of accessing data so that users can spend no effort to learn Grid data tools. Then, the DIRAC component integrates computing resources providing solutions for routing jobs to the proper environment on the basis of the JDL requirements and the user VO permissions and roles. Finally, the gUSE and DCI Bridge components assist the workflow submission: the former provides a set of Web services that interpret and handle a workflow to consume computing resources; the latter, a web application, represents a component between gUSE and the DCIs creating a standard access to Grids, clusters and Clouds.

At the lowest level, the Middleware Resources Layer consists of the Grid and Cloud middleware components to provide physical and virtual resources. The interaction with the Grid services is already in production, whereas the Cloud one is in a prototype version and still in pre-production.

In the following sections we detail the Portal and External AuthZ and AuthN layers and the Portal and External Data/Computing Services layers.

\section{AuthZ and AuthN Details}

The two AuthZ and AuthN Layers (described in Section 3 and shown in Fig. 1) determine the first access to the portal where users through registration must provide the mandatory qualifications to access Grid or Cloud services.

During the authorization phase users must provide the following information: a X.509 certificate issued by a trusted EUGridPMA-member CA, the affiliation to a recognized VO and a trusted IdP. If a user only has a subset of these credentials, the portal could use them to provide the missing ones as described in subsections 4.1 and 4.3. Table 2 shows the credentials combinations required to successfully conclude the registration phase: the $\mathbf{x}$ symbol specifies a missing information; the o symbol states an available information.

The request for a personal certificate and its management (such as storing and renewal) often represent tedious operations that many users wish to avoid. We addressed this issue: by interfacing the portal with an online CA, which provides X.509 certificates to users authenticated by a federated identity management

Table 2 The required credentials for the registration phase

\begin{tabular}{llll}
\hline Case & IDP member & X.509 certificate & VO \\
\hline 1 & $\mathrm{x}$ & $\mathrm{o}$ & $\mathrm{o}$ \\
2 & $\mathrm{o}$ & $\mathrm{x}$ & $\mathrm{x}$ \\
3 & $\mathrm{o}$ & $\mathrm{o}$ & $\mathrm{o}$ \\
\hline
\end{tabular}


system; and by implementing a service to manage these certificates on behalf of the users. Depending on their credentials, users can select whether to upload their certificate or ask for a new one through the portal. The AuthZ portlet is able to manage each step of the registration phase and to perform all the actions according to users' different choices.

During the authentication phase, the portal leverages a federated authentication mechanism based on the Security Assertion Markup Language (SAML) [38] - to offload the portal from managing users' credentials and to exploit a single-sign-on solution. Indeed, the portal trusts all the IdPs belonging to the EDUgain federation [39] that interconnects distributed identity federations around the world; therefore all members of these IdPs can access the portal components by using the credentials contained in their own organization IdP. Since Liferay natively supports the Central Authentication Service (CAS) [40] but not SAML, the portal uses the Casshib [41] software; this enables the CAS server to act as a Shibboleth service provider [42].

The following subsections detail the registration steps for all the cases described in Table 2 and the authentication mechanism. The registration phase collects users' information authorizing them to access Grid and Cloud resources.

\subsection{Registration to the Portal}

Users holding a valid X.509 personal certificate and a VO membership can access the portal: if they are registered in a trusted IdP (case 3 in Table 2), the portal retrieves their personal information from the IdP itself; otherwise (case 1 in Table 2), the portal registers users without IdP in an IdP internal to the portal by using the information got back from their personal certificate. For case 3, the registration phase consists of four steps as shown in Fig. 2: retrieving personal data from the IdP, uploading certificate, declaring the VO membership, and encrypting credentials.

Step 1. The registration page redirects users towards their IdPs. Once validation has succeeded, the portal retrieves personal information, such as first and last name, email address and institute, and stores them in the portal DB.

Step 2. Users have to upload their certificate and provide the passphrase to decrypt it. Hence, the portal gets the passphrase to create two proxy certificates compliant to GSI (Grid Security infrastructure) [43] and RFC 3820 [44] with the same lifetime as the personal certificate. The portal also encrypts the proxies by using a random passphrase, saves the encrypted proxies in a dedicated MyProxy server and finally deletes the personal certificate following the EUGridPMA guidelines on private key protection [45]. Ultimately, the portal inserts the certificate data into the DB.

Step 3. Users have to declare their VOs membership. By the VOMS interfaces the portal assesses the claimed membership and then retrieves their VO information (such as membership, roles and groups) through a secure connection. Users can set their roles and groups for each VO they belong to and set a default VO. Then, the portal inserts the VO information into the DB.

Step 4. Users have to choose a personal passphrase to encrypt the proxy certificates. The portal replaces the old proxy passphrase, randomly generated during the second step, with this new one that is valid until users upload a new certificate. Then, the portal discards the new passphrase and registers the users with the "active" role in the DB.

\subsection{Authentication Mechanism}

The authentication process consists of two steps as shown in Fig. 3: handling single-sign-on validation, and retrieving Grid credentials.

Step 1. The portal through the authentication portlet redirects users to their IdP login page. Then the portal redirects them to the main page where they have access to all the tasks and services made available; otherwise, the portal exposes the registration page by default.

Step 2. Users select a VO from a list containing all the VOs they declared to be part of, and insert the passphrase set during the registration phase (see steps 3 and 4 in subsection 4.1). The portal retrieves a proxy by querying the MyProxy server (long) and saves it setting its lifetime to $\mathrm{N}$ days (users can choose $\mathrm{N}$ in the interval 1-7). The portal then moves the proxy to the MyProxy server (short) and contacts the appropriate VOMS to add extension to this short-lived proxy. 
Fig. 2 The registration steps via Grid credentials: the open arrows describe actions performed by users

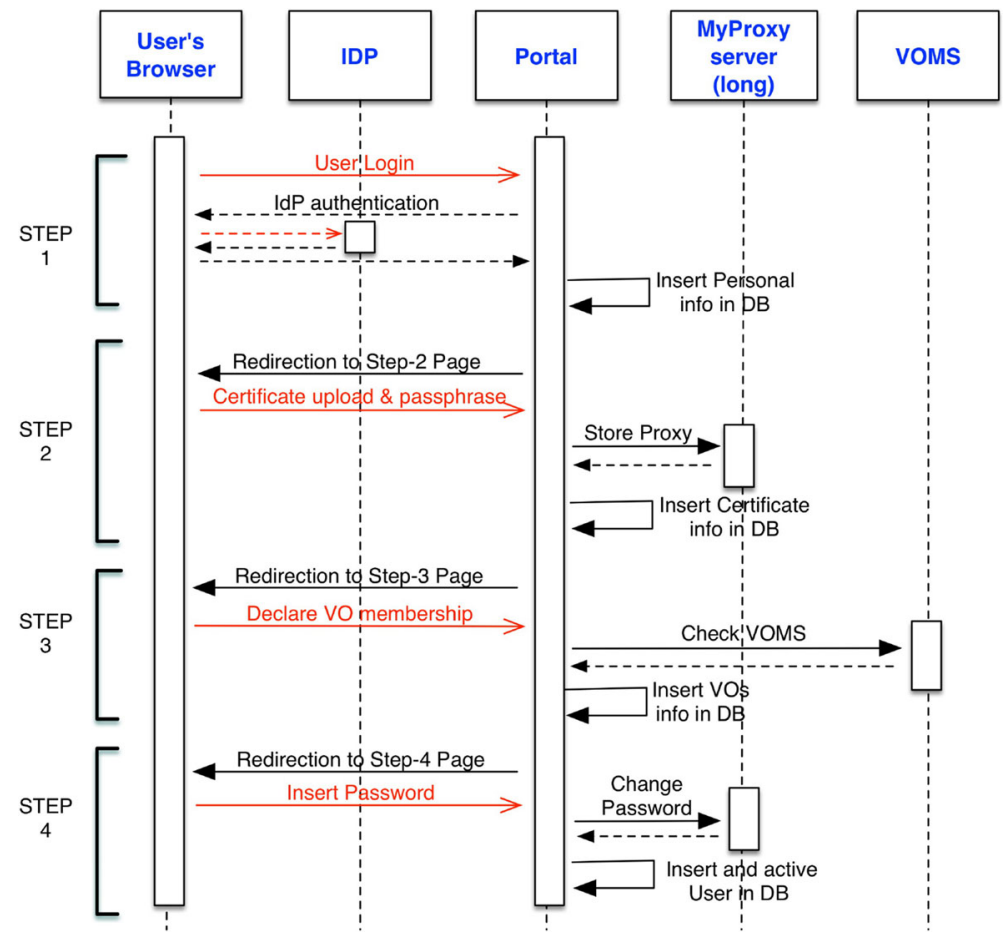

by the portal, which combines the functionalities of an online CA with those of a MyProxy server. In case 2, the $\mathrm{CA}$ relies on pre-existing identity data maintained by users' home institutions, which act in all respects as a trusted registration authority.
Fig. 3 The authentication mechanism: the open arrows describe actions performed by users

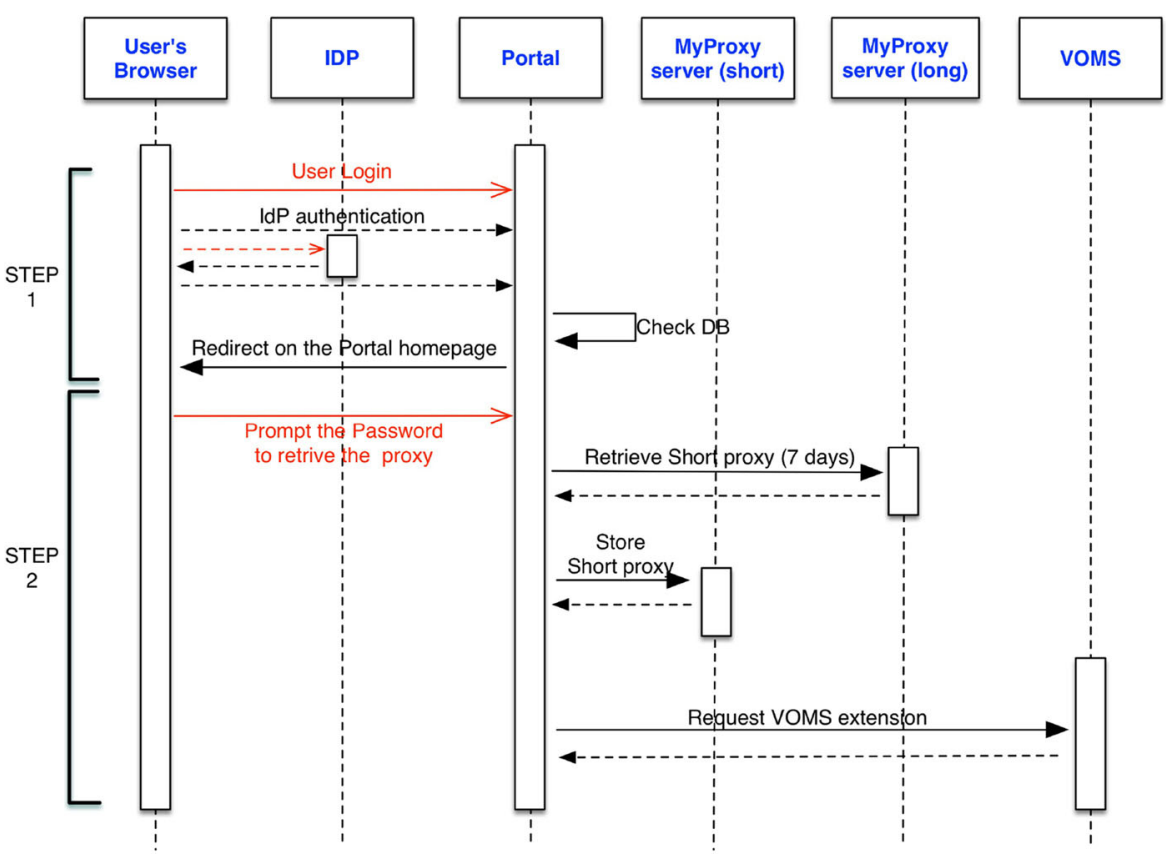


Concerning the online CA, we created a proof-ofconcept to deal with the security level imposed by the EUGridPMA policy. Figure 4 sketches the steps to fulfil the authorization chain from login up to the creation of proxy credentials. The authorization process consists of the following two steps:

Step 1. Retrieving personal data from IdPs is the same as step 1 in Section 4.1. In addition, the portal generates a first token and appends it to the redirected URL towards the CA-bridge endpoint.

Step 2. CA-bridge requires a further authentication because the Shibboleth delegation is still in an experimental phase. This procedure is completely transparent to the users due to the single-sign-on mechanism. The CAbridge makes use of the retrieved information and performs the following operations: it generates a second token; it compares this new token with the one created by the portal; and it verifies the matching. If and only if the two tokens are equal, the CA-bridge triggers the following actions: it asks users to provide a password; it generates a private key and a certificate signing request on behalf of the users; and it sends the request to the CA to have it signed. On receiving the certificate, the CA-bridge stores the long term proxy credential in the MyProxy server (long), destroys the certificate private key, and adds users to a catch-all VO managed by a dedicated VOMS server. Ultimately, the CA-bridge stores certificate and VO peculiar information (e.g. VO name, VO group, and certificate distinguished name) into the portal DB.

By taking all the required steps (retrieving personal data from IdPs and triggering CA-bridge interactions), users get a 13-months lifetime certificate and belong to a catch-all VO without performing any other credential handling.

\section{Data and Computing Services Details}

The Portal and External Data/Computing Services Layers (described in Section 3 and shown in Fig. 1)

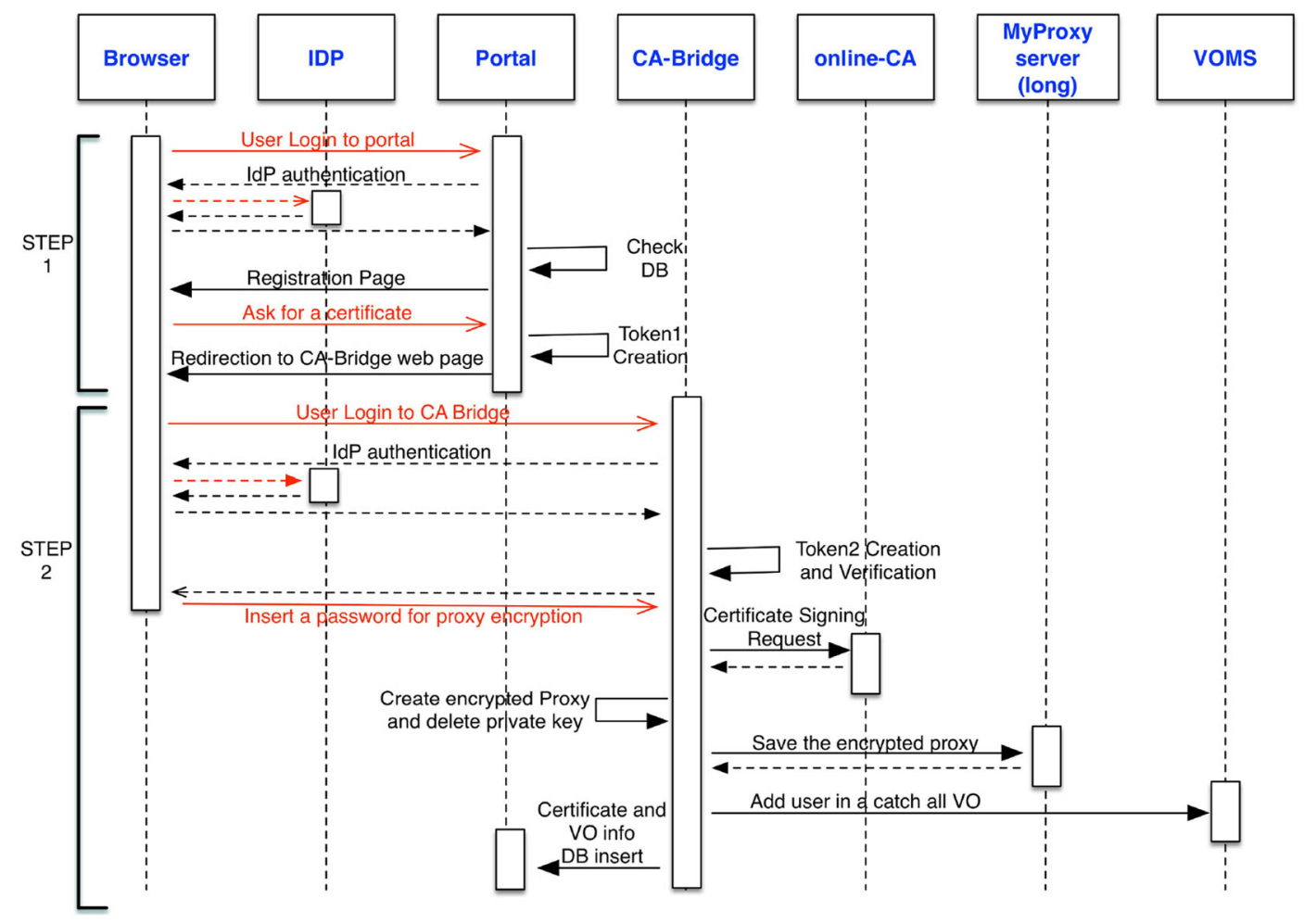

Fig. 4 The registration steps via Online CA: the open arrows describe actions performed by users 
bridge users towards Grid and Cloud resources covering all the requirements described in Table 1. The computing services scenarios comprise the submission of: simple Grid jobs with binaries and input data; workflows with complex use cases; specific applications oriented to custom interfaces; and Cloud provisioning with IaaS (Infrastructure as a Service) allocation. The data service scenario abstracts moving data asynchronously among Grid Storage Elements (SEs) in a drag and drop way. The following subsections detail the foregoing outlines.

\subsection{Simple Grid Jobs}

Users submit jobs by uploading their executables and input files and by adopting JDL to specify the executable parameters. The portal implements this scenario by interfacing the Job portlet with a multi-VOs configured DIRAC server. Despite the DIRAC server provides its own Web interface, at the design level we chose to disable it exploiting the Job portlet that uses the DIRAC command line interface to handle the communication between the portal and the DIRAC server. Consequently, we can both have a single access gate (i.e. the portal) and avoid duplication of features. In addition, the Job portlet allows users to: build their JDL by selecting the correct values from a list of attributes and settings; save JDLs as templates for sharing and reusing purposes; show the list of submitted jobs; monitor the job state during its execution; retrieve the output; resubmit an ended job; log files in the end.

By the DIRAC server, users submit their jobs by exploiting the pilot job mechanism [46] - suitable for massive job submissions. Then the server sends jobs to a central task queue, and later the Grid resources get them asynchronously by executing pilot jobs. The mechanism decouples resource allocation and job management. The DIRAC server also instantiates virtual resources for the job executions discerning among Grid and Cloud resources. It automatically performs this operation according to the job requirements.

\subsection{Workflows}

The workflow submission is a step-by-step procedure for performing complex computations on different resources optimizing the overall task. Each step represents a specific portion of the entire calculation - identifying what we call a job. It can follow conditional constraints according to the evolution of the computation. The adopted acyclic workflow structure can assume a simple or complex form in relation to the addressed problem. Using a subset of WS-PGRADE portlets (workflows creation, submission and management) and wrapping them through the gUSE component the portal allows the support to both types of workflows.

\subsection{Specific Applications}

Some communities may need to submit ad-hoc applications exploiting job and workflow submissions according to VO-specific requirements. In this case, we have adopted the following procedure to adapt existing applications to the Web portal: first, we analysed the portability of the application on the Grid; then, we checked hardware and software requirements (such as RAM, number of CPU cores, and specific libraries) as well as required input and output files, and application parameters needed for retrieving log and output files at runtime. In addition, we created a script that takes care of getting users' inputs, parameters and files, executing the application and retrieving the output produced during the entire computation. This utility script translates the application requirements in the proper execution environment, enabling features such as monitoring the simulation and retrieving runtime logs. If the application required a job submission, we defined an ad-hoc JDL template, included in the job portlet and available to the users for the submission of the job; if the application required a workflow submission, we created a custom workflow in the WSPGRADE framework and we implemented a portlet that exploits such workflow through the ASM APIs provided by WS-PGRADE.

There are several advantages in using a workflow with a custom interface to handle a specific application. The job submission only requires filling a Web form - a simplified access to the Grid. It is straightforward to alter the lifetime of long running Grid jobs by considering the queue maximum wallclock time, and applying a stop-and-go mechanism on the various resources. If a job fails, it is possible to either rely on standard Grid recovery mechanisms or provide the application with a proper tool handling the problem and adopting the appropriate solution. 
Inspecting produced files and monitoring the application are paramount items for long running applications. The job perusal solution (provided by WMS [47]) supports the first item but works only with small-size output files to avoid network overload. To overtake these limitations and to integrate application monitoring into the IGI portal, we developed a new mechanism, called application progress monitoring, exploiting Grid SEs and Storage Resource Manager (SRM) command line interfaces (CLIs) to make temporary and partial output files available to be inspected at runtime. The adopted SRM CLIs mechanism copies selected files from the computing resource where the job is physically running to a Grid SE that stores the files. Then users can directly access these SE files via the job or ASM portlet.

At last to simplify to the user the choice of input files and output destinations, these applicationspecific portlets interact with the Grid data management services through their specific utilities.

\subsection{Cloud Provisioning}

Some communities may need to run their applications exploiting the Cloud paradigm. The portal implements this scenario by interfacing a Cloud portlet with a set of services that supply resources according to the IaaS provisioning model. The services, fed with a pre-built configuration file, can interact with various IaaS Cloud providers exploiting the benefits offered by existing Cloud platforms such as WNoDeS [48], OpenStack [49] and Opennebula [50]. The developed portlet exposes a Web interface for each service simplifying users' tasks to create and manage new instances.

To instantiate new virtual machines (VMs), users have to upload their own SSH public key [51], or generate a SSH private and public key pair through the portal. This is necessary to allow logging into a VM with root privileges without requiring any password. The keys generated by the portal can be retrieved by users in a secure way. Users can then create new VM instances choosing from a list of images preloaded in a repository. For each image it is possible to select the size of the cloud environment (defined according to the number of cores, memory and disk size) and how many instances have to be created at a time; each image has a range size that depends on the Cloud platform it belongs to. As soon as users create new instances, a list of their VMs is displayed together with information such as architecture, size and status. By selecting the instance name, users are automatically logged into the VM with root privileges through a Web terminal [52] that is part of the portal. The portal lets users select VM images offered by different Cloud providers via a repository, which access depends on the resource providers internal policies.

The portal Cloud feature is in a prototype version but close to a production-quality solution. We presented at the EGI Community Forum 2013 [53] its successful validation by the World Wide eInfrastructure for NMR (WeNMR) [54] virtual research community to address its CING use case (fully detailed in [55]). The prototype uses the Worker Nodes on Demand Service (WNoDeS) Cloud command line utilities - developed in the context of the EGI Federated Cloud task force [56] to simplify the handling of further Cloud needs and site peculiarities - leveraging the strength of different Cloud platforms such as OpenStack and WNoDeS. Furthermore, it interacts with the Stratuslab Marketplacebased repository [57] to get preloaded images information. Figure 5 shows the Cloud portlet interface for choosing a custom WeNMR image.

\subsection{Data Management}

In a standard Grid environment users may benefit from a set of command line tools to perform data management tasks such as copying files on a Grid SE, registering files in a LCG File Catalogue (LFC) [58], and replicating files on other Grid SEs. To save learning effort, we designed and implemented a data management service [59] sketched in Fig. 6. The service includes several elements intercommunicating in a secure way.

The Data Mover component controls and manages every step of the file transfer operations: uploading and downloading files. It controls data transfers through an external storage service - composed of a set of Storage Resource Manager (StoRM)-based portal SEs [60] acting as a cache memory for the files - until they are transferred to or downloaded from a Grid SE. The Data Mover is a Web Pydiobased data management service that implements and extends the functionalities of the Grid data management command line tools, exposing a Web interface that manages either Grid files or some other types of 
Fig. 5 The Cloud portlet interface for choosing a custom WeNMR image preloaded from the Stratuslab Marketplace repository

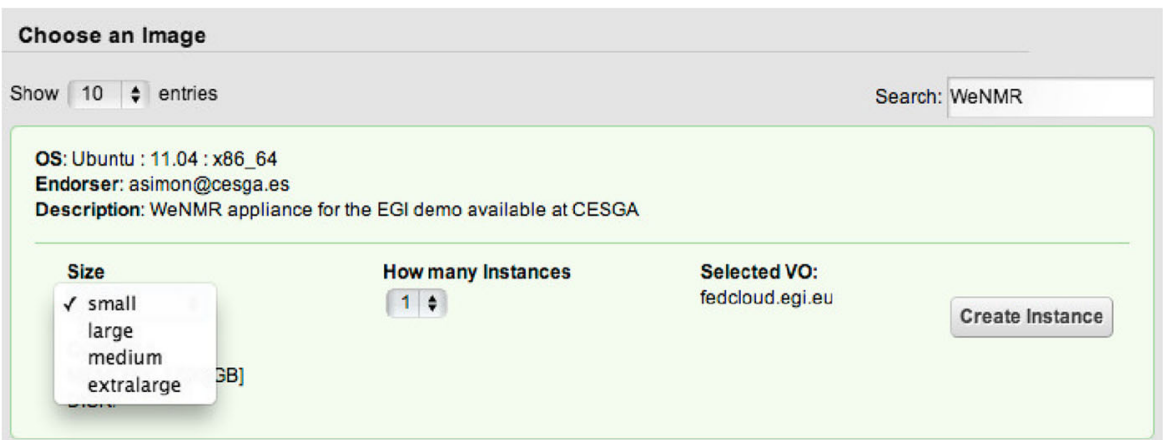

files. We developed a plug-in for handling data that by the IGI portal allows users to browse the content of the VO file catalogue and to perform operations on either the logical data (affecting the information contained in the catalogue) and/or the physical files (involving the files physically stored in the SEs). The LFC contains the mapping of a generic file name, the Logical File Name (LFN), to one or more physical locations of a file on the Grid. Each file is univocally identified by a Global Unique Identifier (GUID), a non-humanreadable fixed-format string that identifies an item of data. Each file can be replicated in different locations and each replica is identified by a Site URL (SURL) or Physical File Name (PFN). Table 3 details the possible operations performed on data.

To upload a local file to a SE, users can select the SE from a list of possible destinations dynamically produced according to the available storage space and users' VO. When users select a specific SE, the system tries to upload the file to that destination; on failure, it randomly tries other SEs from the same list. If users need to download one or more files from the Grid, they can choose to copy it/them either to a local destination or to an external server. To avoid transferring big files to users' local space, we set a limit of five Gbytes to the maximum file size to be downloaded locally. The supported protocols for the data transfer to external servers are SFTP, FTP(S), and HTTP(S). To perform the operations shown in Table 3 on logical and physical data, the Data Mover directly communicates with the standard Grid services, such as LFC and SEs. Therefore the data management service architecture allows a complete decoupling of the Web interface, the control service (Data Mover) and the physical storage
Fig. 6 The IGI Portal data management service architecture

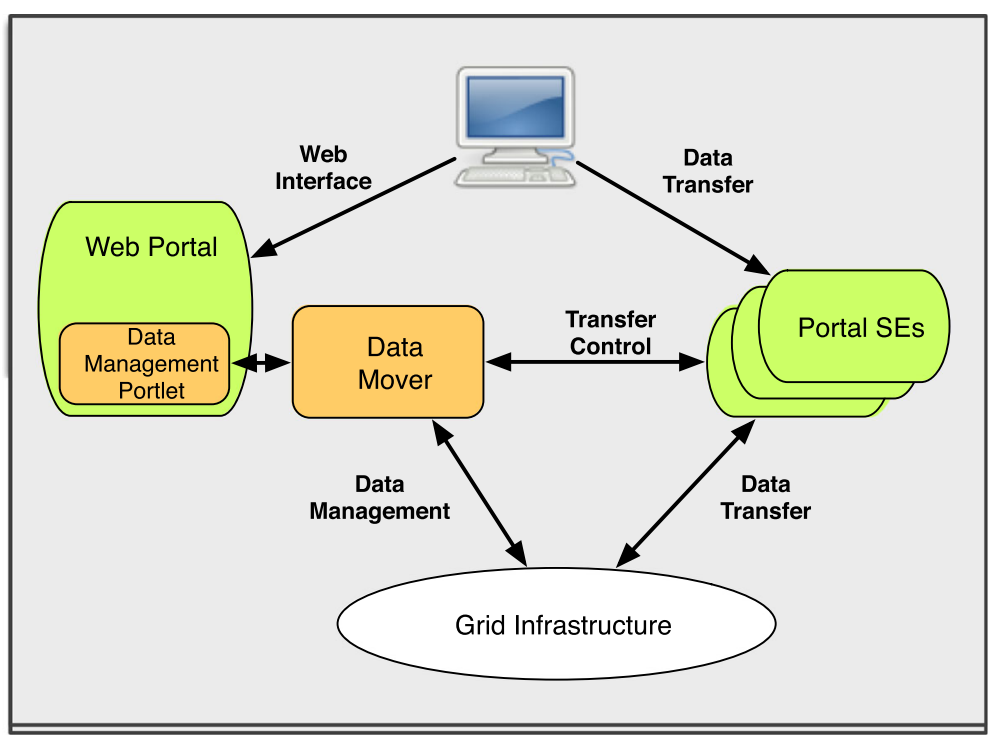


Table 3 The possible operations performed on data

\begin{tabular}{ll}
\hline Data Types & Operations \\
\hline & Creating a new folder; \\
& Deleting an empty folder; \\
& Renaming a folder or file (changing the LFN); \\
Logical Data & Moving a folder or file (changing the LFN); \\
& Getting detailed information about a file \\
& (LFN, GUID - Global Unique Identifier, \\
& list of replicas, owner, ACL); \\
& Sharing a file with other portal users. \\
& Replicating files on different storage elements; \\
& Downloading files. \\
Physical Data & Deleting files; \\
Logical and & Uploading files. \\
Physical Data &
\end{tabular}

(portal SEs). In addition, the number of portal SEs can be extended, if necessary, to provide final users with a reliable and fast data transfer in case of a portal bottleneck (e.g. many users and many transfers).

\section{Use Cases}

The Italian and European Grids were initially created to satisfy the computational and storage requirements of the high energy physics communities applications that had driven the shaping of the infrastructure. In the last decade, as the result of the participation to various Italian and European projects (i.e. the EGEE [61] series, Grid.it, INFNGrid and EGI-InSPIRE [62]), the same infrastructure [61] has increasingly supported new user communities belonging to various scientific domains such as computational chemistry, bioinformatics, astronomy and astrophysics, earth science, mathematics, and engineering. These new communities differ in computational and storage requirements, composition, computational experience and size. Porting their applications and creating Grid-based computing models (that fit their needs) are sometimes hard to realize. Dedicated support, in fact, assists the development of high level Web interfaces addressing their computing models to hide the Grid complexity. We also designed the IGI Web portal to create applicationspecific Web interfaces enabling users to access the standard functionalities of the Grid infrastructure and to run their computational applications by hiding the inner complexity. For this reason, we developed a set of specialized portlets that run specific use cases based on commonly adopted applications. Table 4 lists the application portlets currently hosted by the IGI portal, where the computational requirements are related to the specific use cases. It also shows the data requirements per single job (only for the most demanding use cases) and the exploited IGI portal features. It is important to point out that all the implemented solutions are the result of a collaboration between the IGI unit dealing with user support and the user representatives who drove their design and validated their implementations.

The following subsections describe two of the interfaces developed so far: the Fluka portlet [63] that is an example of a pure high throughput use case (i.e., many independent, single core jobs); and the portlet for Theoretical Physics applications that is an example of a use case that needs long parallel jobs and checkpointing.

\subsection{Fluka MonteCarlo Simulation}

Porting an application to the Grid environment means executing code on multiple Grid resources with different input files simultaneously. As, in general, these simulations can run independently from each other, they can take advantage of their distribution on a wide computing infrastructure. This is the case of the Fluka code resulting from a collaboration between INFN and CERN - a general Monte Carlo tool for the calculations of particle transport and interaction with matter. It has a wide range of applications in fields such as cosmic ray physics, particle physics, and neutrino physics. We compiled Fluka as a static binary by using the gfortran compiler and other open source libraries. The static compilation of the package ensures that the program is binary compatible with the Grid computing resources, preventing the incompatibility errors associated with the usage of dynamically loaded libraries.

The sequential Fluka program follows the so-called parameter study (or "parameter sweep") approach to use the Grid. In our case, the various Fluka jobs work with different seeds for a different set of initial conditions. For the above mentioned reasons the Fluka application portlet makes use of the DIRAC framework to perform a massive job submission. In such way a simple and intuitive interface assists users in 


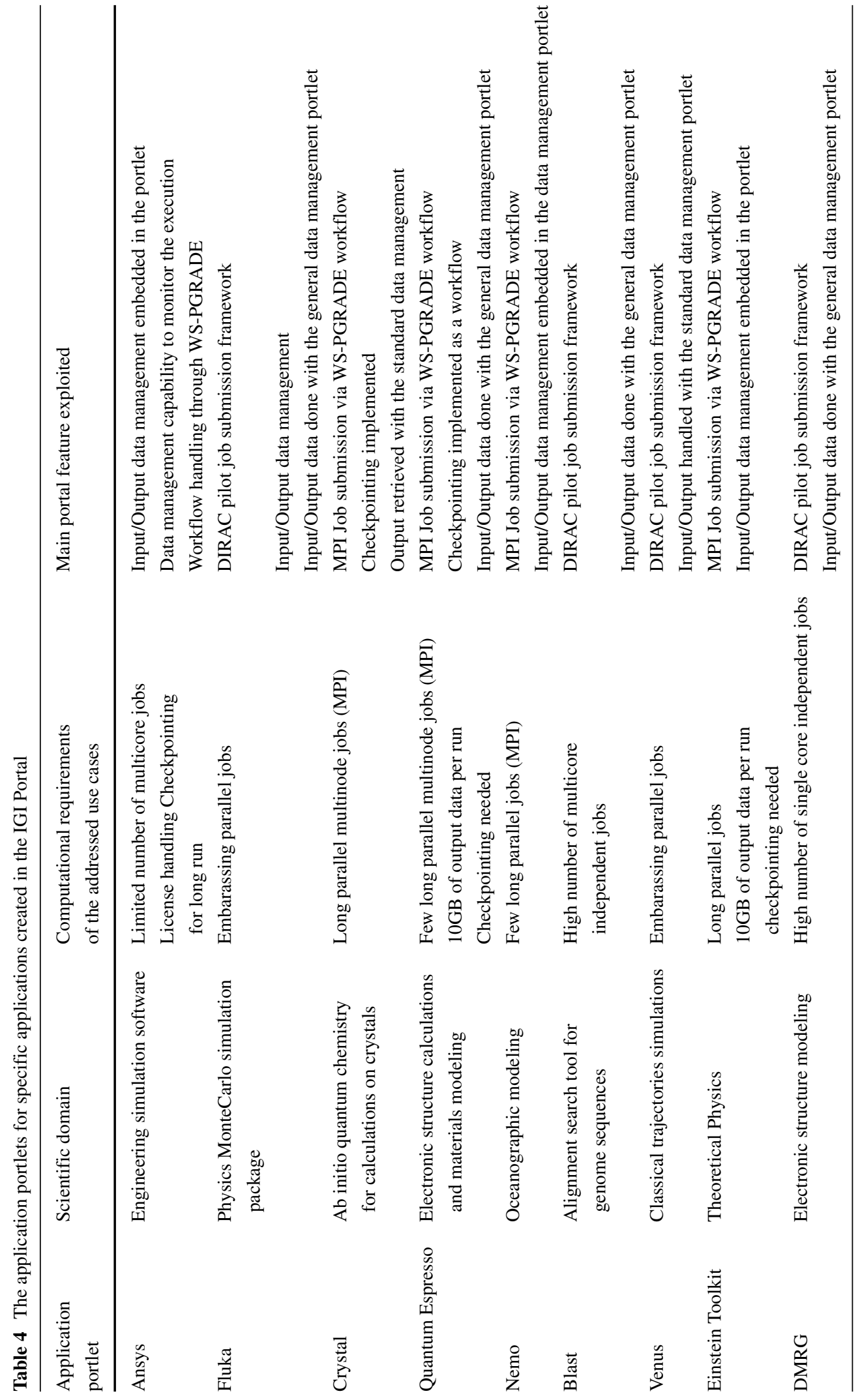


the management of a multitude of sequential jobs from submission up to output retrieving. We tuned the portlet for a Fluka-based use case provided by the SPES [64] experiment community, which aimed at evaluating the low-energy neutrons treatment and their related gamma emission inside the experimental apparatus. Within this particular use case, users submitted a set of 1000 jobs and, after about 32 hours, collected the results from 847 successful job submissions for a grand total of about $10.1 \mathrm{k}$ CPU-hours. The remaining jobs failed due to hardware and/or software related problems that need to be investigated. The average run time of successful jobs was about 12 hours.

Using the Fluka portlet (see Fig. 7), users can set the needed fields such as the name of the submitted jobs to distinguish each set of submission; the input file already stored in the SE and selectable from the user by using a dropdown menu; the output folder in the LFC directory where the output will be stored; and the number of parameter sweep jobs to be run in a single submission.

\subsection{Theoretical Physics Applications}

In the theoretical physics field, applications require both serial jobs-based computation and High Performance Computing (HPC) resources ranging from large computing clusters up to specialized supercomputers, according to the problem size. Among the latter, we can mention lattice quantum chromodynamics, fluid dynamics and numerical relativity.

Building on resources available on IGI, the INFN theoretical physics community (Theophys VO) has recently started a project [65] aiming at fulfilling the requirements of medium-sized HPC applications. This project is achieving important results for the Theophys community, such as the deployment of a common infrastructure for parallel applications that shares the same platform with the other Grid resources available for serial jobs. On the other hand, there are aspects that still have to be improved such as the usability of the infrastructure. For this reason the Theophys community undertook a collaboration with the IGI Web portal
Fig. 7 The Fluka application portlet
Home
Ansys
Fluka
Storage
My Data
Calendar
Wiki

Hi Marco, your active Vo: gridit Get Credentials
My Jobs

JDL

Job Name

FLUKA_Job_\%s

Help: The flag "\%s" indicates the number of the job. Useful for parametric jobs

vo

gridit (Default) $\rightleftharpoons$

Input

$+1$

Output Folder

Same of inputs

Number of Jobs

1

Help: Used for parametric submission. Is the number of jobs that you want to submit. Its value can be equal or less then the number of input files included in the input tar archive.

Start Number

Help: Is the first job number used to start the submission. More

Submit Job List 
developers, aiming at supplying a customized portlet see Fig. 8) conceived to ease the parallel job life cycle in all its stages:

- Creation/submission: The data management for these applications is based on two Grid directories used to upload respectively the executables and the input files. Three dedicated drop-down menus support users in the executables uploading, files uploading and resources selection (defined by a community administrator). To create a new job, users only have to select files and resources from the proper menus and to provide execution details (such as executable arguments, output filename, and HPC computational environment)

- Monitor: the portlet makes available standard output and standard error files; since they are periodically updated, users can check the job state at any time;

- Output retrieval: after the job completion, the job packs the whole execution directory into a tar archive and copies it back in the previously selected Grid directory. This feature is particularly useful for checkpointing and debugging purposes.

Among the different parallel applications used by the Theophys community, we selected the Einstein Toolkit [66] - an open software providing the core computational tools needed by relativistic astrophysics, i.e. to solve the Einstein's equations coupled to matter and magnetic fields. The toolkit needs parallelization to speed up the computation and to distribute the memory allocation of the evolved variables among the available resources. The code uses an hybrid
MPI/OpenMP [67, 68] environment, so it requires entire computational nodes which number depends on the lattice size. Another important requirement of this application is the need for checkpointing that is often used for writing large data-output (of the order of 10 GBytes). The Theophys user community performed a set of production submissions (requiring 32 whole nodes, long execution time and checkpoints) to test the behaviour of the portlet. The runtime monitoring of standard output and standard error proved to be useful in different stages of the job life-cycle, while the data management portlet performed the retrieving and the management of checkpoints among different sites.

\section{Conclusions and Future Activities}

This paper describes a Web portal targeted at scientific communities making use of distributed resources. Through the portal, indeed, users can access Grid and Cloud resources, submit jobs or complex workflows with dependency-related applications, move data to and from the Grid resources, and finally access specific applications through custom interfaces. Furthermore, the portal modular architecture and the use of widely adopted frameworks make the integration of new services easy by developing appropriate portlets.

This paper also shows the benefits offered by the IGI portal by describing some relevant use cases. The implemented use cases show that various advantages can be obtained from the heterogeneous nature of the EGI Grid production infrastructure when porting scientific applications on distributed platforms. These
Fig. 8 The Theophys application portlet

$\begin{array}{llllll}\text { Welcome Job Workflow THEOPHY Storage My Data Help } & \end{array}$

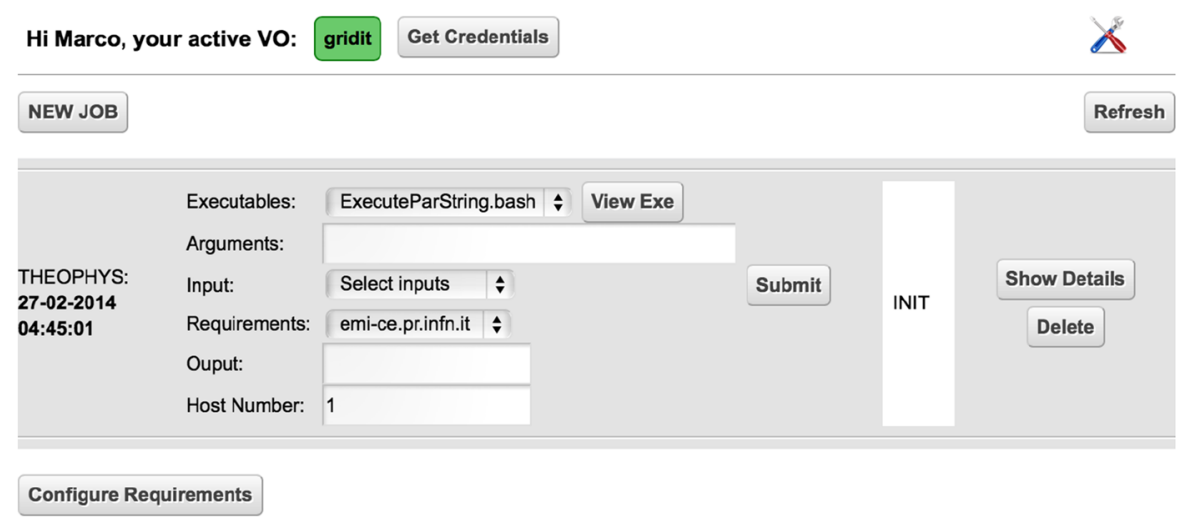


include the possibility of partitioning large calculations into smaller ones and selectively distributing segments on different machines to gain efficiency as well as feasibility. This makes the work performed in the application porting phase (see Section 5.3) of more general use by providing a reusable example for other domain-specific applications.

In the future work, we are planning to integrate additional scientific applications into the portal, also targeted to attract new user communities, on different operating system flavours as well as hardware platforms. The current portal implementation mainly focuses on access to resources belonging to the Grid world. However, we have planned to make a deeper integration with Cloud resources: extending the Cloud high-level interface for the direct provisioning of SaaS (Software as a Service) solutions; implementing a tighter integration with Cloud frameworks such as OpenStack or OpenNebula; supporting data transfer across Grid and Cloud resources; enhancing the reporting for status errors linked to storage provisioning; improving support for scalable and distributed Grid and Cloud storage systems; extending the Cloud interface to support non-Linux systems.

Acknowledgements The research leading to the results presented in this paper has been possible thanks to the Grid resources and services provided by the European Grid Infrastructure (EGI) and the Italian Grid Infrastructure (IGI). We express our appreciation to the late Dr. Valerio Venturi.

\section{References}

1. Kesselman, C., Foster, I., Tuecke, S.: The anatomy of the grid: enabling scalable virtual organizations. Inter. J. Supercom. Applic. 15(3), 200-222 (2001)

2. WLCG Website. http://wlcg.web.cern.ch

3. Wengler, J.C.: How grid computing helped CERN hunt the Higgs. www.istw.org (2012)

4. NERSC science gateways. http://www.nersc.gov/users/ science-gateways

5. XSEDE website. https://www.xsede.org/gatewaysoverview

6. Ardizzone, V., Barbera, R., Calanducci, A., Fargetta, M., Ingrà, E., Porro, I., La Rocca, G., Monforte, S., Ricceri, R., Rotondo, R., Scardaci, D., Schenone, A.: The DECIDE science gateway. J. G. Comp. 10(4), 689-707 (2012)

7. What is a science gateway? http://sciencegateways.org/ what-is-a-science-gateway

8. The Grid Chem project. https://www.gridchem.org

9. SCI-BUS project. Science gateways summary. http://www. sci-bus.eu/science-gateways
10. Barbera, G., Donvito, A., Falzone, J.J., Keijser, G., La Rocca, L., Milanesi, G.P., Maggi, R., Andronico, G., Vicario, S.: A grid portal with robot certificates for bioinformatics phylogenetic analyses. Con. Comp: Prac. Expe. 23(3), 246-255 (2011)

11. EUGridPMA guideline on approved robots. http://www. euGridpma.org/guidelines/robot/

12. Kacsuk, P., Farkas, Z., Kozlovszky, M., Hermann, G., Balasko, A., Karoczkai, K., Istvan, M.: WS-PGRADE/ gUSE Generic DCI gateway framework for a large variety of user communities. J. G. Comp. 9(4), 479-499 (2012)

13. IGI website. www.italianGrid.it/about

14. Basney, J., Humphrey, M., Welch, V.: The MyProxy online credential repository. Softwa. Pract. Experience 35(9), 801816 (2005)

15. Tsaregorodtsev, A., Bargiotti, M., Brook, N., Ramo, A.C., Castellani, G., Charpentier, P., Cioffi, C., Closier, J., Graciani, R., Kuznetsov, G., Li, Y.Y., Nandakumar, R., Paterson, S., Santinelli, R., Smith, A.C., Miguelez, M.S., Gomez, S.: DIRAC a community grid solution. J. Phys. Conf. Ser. 119(062048), 2008 (2008)

16. gUSE website. guse.hu

17. Thomas, M., Mock, S., Dahan, M., Mueller, K., Sutton, D., Boisseau, J.R.: The GridPort Toolkit: a system for building Grid portals, pp. 216-227. IEEE Xplore Digital Library, San Francisco (2001)

18. Calzolari, F., Licari, D.: Proxy dynamic delegation in grid gateway. Proceedings of Science (PoS), (PoSISGC 2011 \& OGF 31027) (2011)

19. Gannon, D., Alameda, J., Chipara, O., Christie, M., Dukle, V., Fang, L., Farrellee, M., Kandaswamy, G., Kodeboyina, D., Krishnan, S., Moad, C., Pierce, M., Plale, B., Rossi, A., Simmhan, Y., Sarangi, A., Slominski, A., Shirasuna, S., Thomas, T.: Building grid portal applications from a web service component architecture, vol. 93, pp. 551-563. EEE Xplore Digital Library (2005)

20. Murri, R., Kunszt, P.Z., Maffioletti, S., Valery T.: GridCertLib a single sign-on solution for grid web applications and portals. J. G. Comp. 9(4), 441-453 (2011)

21. CILogon website. www.cilogon.org

22. Cilogon ca levels of assurance. http://ca.cilogon.org/loa

23. EUGridPMA website.www.euGridpma.org

24. Incommon website. http://www.incommon.org/federation

25. Terena website. http://www.terena.org/activities/tcs

26. EJBCA website. www.ejbca.org

27. Pydio website. pyd.io

28. own Cloud.org website. owncloud.org

29. elFinder website. elfinder.org

30. jQuery website. https://github.com/blueimp/jQuery-FileUpload/wiki

31. LHCb website. http://lhcb-public.web.cern.ch/lhcb-public/

32. Liferay website. www.liferay.com

33. JSR 168: portlet specification, java community process http://www.jcp.org/en/jsr/detail=168 (2005)

34. JSR 286: portlet specification 2.0 , java community process http://www.jcp.org/en/jsr/detail=286 (2008)

35. MySQL website. www.mysql.com

36. Alfieri, R., Cecchini, R., Ciaschini, V., dell' Agnello, L., Frohner, A., Lorentey, K., Spataro, F.: From Gridmapfile to VOMS: managing authorization in a grid environment. Futur. Gener. Comput. Syst. 21(4) (2005) 
37. TAGPMA, profile for member integrated X.509 credential services with secured infrastructure. http:// www.eugridpma.org/guidelines/MICS/IGTF-AP-MICS-1. 2-clean.pdf

38. Hardjono, T., Klingenstein, N.: SAML V2.0 Kerberos Web Browser. In: PSSO Profile Version 1.0 Technical report OASIS (2010)

39. eduGAIN website. www.geant.net/service/eduGAIN/Pages/ home.aspx

40. CAS website. http://www.jasig.org/cas

41. Casshib website. https://code.google.com/p/casshib

42. Shibboleth website. http://shibboleth.net/products/serviceprovider.html

43. Foster, I., Kessekan, C., Tsudik, G., Tuecke, S.: A security architecture for computational grids. In: The 5th ACM conference on computer and communication security (1998)

44. RFC 3820. http://www.rfc-base.org/rfc-3820.html

45. EUGridPMA guidelines on private key protection. http:// www.eugridpma.org/guidelines/pkp/

46. Sfiligoi, I., Tiradani, A., Holzman, B., Bradley, D.C.: The Glidein WMS approach to the ownership of system images in the cloud world. In: Leymann, F., Ivanov, I., Sinderen, M.v., Shan, T. (eds.): CLOSER, pp. 443-447 (2012). SciTe Press

47. Cecchi, M., Fabio, C., Dorigo, A., Ghiselli, A., Giacomini, F., Maraschini, A., Marzolla, M., Monforte, S., Pacini, F., Petronzio, L., Prelz, F.: The glite workload management system. In: GPC of lecture notes in computer science, vol. 5529, pp. 256-268. Springer (2009)

48. Salomoni, D., Italiano, A., Ronchieri, E.: WNoDeS, a tool for integrated grid and cloud access and computing farm virtualization. In: Journal physics: Conference Series 3315 (Computing Fabrics and Networking Technologies) (2011)

49. OpenStack website.www.openstack.org

50. OpenNebula website opennebula.org

51. Barrett, D.J., Silverman, R.E., Byrnes, R.G.: SSH. In: The secure shell the definitive guide O'Reilly Media (2005)

52. GateOne website. http://liftoffsoftware.com/Products/ GateOne
53. EGI Community Forum 2013 website. cf2013.egi.eu.

54. WeNMR website. www.wenmr.eu

55. Ronchieri, E., Verlato, M., Salomoni, D., Torre, G., Italiano, A., Ciaschini, V., Andreotti, D., Pra, S.D., Touw, G.V.W.G., Vuister, G.W.: Accessing Scientific Applications through the WNoDeS Cloud Virtualization Framework. In: PoSISGC 2013029 (2013)

56. EGI Federated Clouds Task Force website. https://wiki.egi. eu/wiki/Fedcloud-tf:FederatedCloudsTaskForce

57. Konstantinou, I., Floros, E., costs, N.K.: Public vs private cloud usage the StratusLab case. In: The 2nd International Workshop on Cloud Computing Platforms CloudCP'12. ACM, Bern (2012)

58. Baud, J.P., Lemaitre, S.: The LCG file catalog (LFC). Technical report. In: CERN (2005)

59. Bencivenni, M., Brunetti, R., Caltroni, A., Ceccanti, A., Cesini, D., Di Benedetto, M., Fattibene, E., Gaido, L., Michelotto, D., Misurelli, G., Venturi, V., Veronesi, P., Zappi, R.: A web-based utility for Grid data management. In: PoS(ISGC 2012004) (2013)

60. Magnoni, L., Zappi, R., Ghiselli, A.: StoRM: a Flexible Solution for Storage Resource Manager in Grid. In: The IEEE 2008 Nuclear Science Symposium (NSS-MIC 2008), pp. 19-25. IEEE Computer Society., Dresden (2008)

61. EGEE website. public.eu-egee.org

62. EGI-InSPIRE website. https://www.egi.eu/about/egiinspire

63. FLUKA website. http://www.fluka.org/fluka.php (2014)

64. Spes website. http://web.infn.it/spes

65. Alfieri, R., Arezzini, S., Ciampa, A., De Pietri, R., Mazzoni, E.: HPC on the Grid the Theophys experience. J. Grid Compu. 11, 260-265 (2013)

66. Löffler, F., Faber, J., Bentivegna, E., Bode, T., Diener, P., Haas, R., Hinder, I., Mundim, B., Ott, C., Schnetter, E., Allen, G., Campanelli, M., Laguna, P.: The Einstein toolkit: a community computational infrastructure for relativistic astrophysics. Class. Quant. Grav. 29 (2012)

67. MPI Forum. www.mpi-forum.org

68. OpenMP website. www.openmp.org 